\title{
Large Eddy Simulation of flow around a single and two in-line horizontal-axis wind turbines
}

\author{
Amin Allah Veisi ${ }^{a}$, Mohammad Hossein Shafiei Mayam ${ }^{\mathrm{b}, *}$ \\ ${ }^{\text {a }} \mathrm{PhD}$ student,Department of Mechanical Engineering, University of Sistan and Baluchestan, Zahedan, \\ Iran. amin_veysi@yahoo.com \\ ${ }^{\mathrm{b}}$ Assistant Professor, Department of Mechanical Engineering, Bozorgmehr University of Qaenat, Iran, \\ shafiei@buqaen.ac.ir
}

\begin{abstract}
A Large Eddy Simulation (LES) has been employed in order to study the flow field in a single-wind turbine and in two in-line wind turbines. The present study focuses on the flow around a horizontal axis wind turbine in a virtual wind tunnel. The anisotropic residual stress tensor is driven by the Smagorinsky model. The results are consistent with experimental data presented in literature. Streamwise velocity is increased and cross stream velocity is decreased as wake moves in downstream direction. A faster rate of wake recovery is seen for the two in-line setup. The results reveal that turbulence intensity is increased by increasing the downstream distance and two in-line turbines show greater intensity. Wind turbine performance can be affected by the turbulent structures. If this phenomenon occurs, information about turbulent structures would be useful in order to investigate the effect of turbulent structures on wind turbine performance. As a result, we aim to reveal the effect of turbulent structures, by using the $\lambda_{c i}$ technique in this study, and to investigate the performance of wind turbines in different conditions. Furthermore, the effects of blade rotation direction are studied in this paper. It is concluded that wind turbine efficiency is increased by $4 \%$.
\end{abstract}

Keywords: Large Eddy Simulation, Wind turbine, Smagorinsky model, Turbulence intensity

\section{Introduction}

Extreme and rapid developments in science, technology, and in the human population have caused a growth in energy consumption. The high levels of energy consumption around the world, global warming, and concerns over the depletion of fossil resources have encouraged the use of renewable 
energy as an alternative energy source. As a renewable energy source, wind energy is of great importance. The capacity of wind power around the world is much greater than the world's energy consumption [1]. It can be seen therefore, that the increasing demand for energy in recent years has resulted in the rapid development of wind turbines and wind farms around the world. The wake behind wind turbine is divided into two regions: near wake and far wake [2]. The near wake is approximately one rotor diameter downstream of the wind turbine. Here, the effect of the blade geometry and vortices that are shed from each blade is prevalent. Tip vortices are formed due to the pressure difference between the two sides of the blade. The path line of these vortices is helical and is induced by the rotating blades. Tip vortices are also responsible for noise generation and blade vibration [3]. There is not enough information available about the behaviors of vortices and their effect on downstream wind turbines and therefore, it is difficult to predict the fatigue loads of the downstream wind turbines. Optimization of the wind turbine distances is possible by using the turbulence structure behaviors. In other words, the fluid flow effects on the wind turbines could be studied from the turbulence structure information available. This investigation helps us to optimize the wind farms in order to increase their performance. The far wake is the region beyond the near wake, where the effects of wind turbine geometry are less important. The downstream wind turbine is affected by the wake of an upstream wind turbine. In fact, the downstream wind turbine experiences different conditions from a single wind turbine, which is why the study of such flow is important. The downstream wind turbine harvests at a lower velocity and has higher turbulence intensity. On the other hand, acquired power via a wind turbine is proportional to the cube of the velocity, which is why less power is obtained from a downstream wind turbine than a single-wind turbine. For these reasons, the study of wake development behind wind turbine blades is important to minimize the effects of wake interaction and increase the performance of the wind turbine [4]. Moreover, in recent studies the intermittent turbulent character of atmospheric wind speed [5] is transferred to the power output from wind farms [6, 7]. In other words, the power output of wind farm possesses intermittent and multifractal properties [5, 6]. Calif and Schmitt proposed methodologies to describe the intermittency of wind power fluctuations at all intensities [6].

Although the tip local Reynolds number is $\operatorname{Re}_{c}^{t i p} \approx 10^{5}$ for the upstream turbine, a full-scale wind turbine has a much higher value (at least one order of magnitude higher). It should be noted that the tip local Reynolds number has significant effects on the functional characteristics of wind turbines. For example, the maximum power generated by the wind turbine working at a low Reynolds number, is less than a similar wind turbine that works at a high Reynolds number [8]. Medici and Alfredsson [9] suggested that vortex shedding and turbulence structures in the near wake of wind turbines are independent of the tip local Reynolds number. Tip vortices are distinct features of horizontal-axis wind turbine wakes. They are shed from the tip of each blade and are transported in the downstream until they eventually dissipate. The fatigue load of downstream wind turbines is affected by turbulence intensity, tip vortex frequency and the dissipation distance of these vortices in the wake region. Hence, the study of wake development, turbulence intensity, and turbulence structures is very important to acquire the highest efficiency in wind farms. Therefore, numerous numerical and laboratory studies have been conducted in order to investigate the wake behind a horizontal-axis wind turbine [10]. 
Recent works have commonly used Reynolds-averaged Navier-Stokes equations that yield to a timeaveraged velocity field. Instantaneous velocity fields are needed to develop a better understanding of such flows. LES has been employed by many researchers to overcome this issue.

LES has been used to acquire detailed fluid flow results in the wind turbine wake region. Troldrborg [11] used actuator line technique and LES turbulence model to solve Navier Stokes equations. He showed, the wake becomes fully turbulent if the ambient turbulence is applied. Numerous numerical studies have been conducted to predict the wake deficit in the downstream direction of a horizontal axis wind turbine [12-14]. Jang-Oh Mo et al. [15] used LES to study the effects of wind speed changes on the wake instability of a wind turbine NREL Phase VI inside a virtual wind tunnel. They suggested a new definition for the distinction between the near and far wake. Jang-Oh Mo et al. [16] also performed another study to investigate the wake behind the wind turbine using LES. Their results showed the formation of two pairs of counter-rotating vortices, which caused a gradual increase in turbulence intensity in the far wake region. Porte'-Agel et al. [17] studied the atmospheric boundary layer flow and its interactions with wind turbines. The results can be used to increase the energy output efficiency and lifetime of wind turbines. Stevens et al. [18] performed LES in order to study the effect of wind turbine row alignment and wind farm length with respect to the incoming flow direction. Their results illustrated that a staggered configuration would not necessarily be the best configuration for a wind farm. Norris et al. studied [19] the effect of the atmospheric boundary layer passing through a wind farm by using LES. They also studied the effects of wake downstream of the wind turbine. Their results revealed that the spread of the wake model predictions is considerable even for a single-wake case. Thus, they suggested further works be conducted for multiple wake cases. Jimenez et al. [20] performed LES to predict the details of wake in the downstream side of a wind turbine. They proposed that wake details and turbulence structures were studied in future works. Li et al. [21] studied the effect of turbulence intensity and wind shear on the power characteristics of a HAWT. Their results showed that the power coefficient was heavily dependent on the blade pitch angle and yaw angle. Li et al. [22] focused on the effect of turbulence on the dynamic stall characteristics in a HAWT. They found that, compared to the static state, the lift coefficients of the dynamic state had larger values than those in the increasing direction of angle of attack. Giahi, and Dehkordi [23] studied the effects of scaling on wind turbine characteristics, including shaft torque, thrust, and aerodynamic forces on the blade. Their results revealed that the torque increases with the cube of change in rotor diameter, whereas the thrust value and aerodynamic forces rise with the square change in diameter. Zhong et al. [24] used LES of a wind turbine at different rotational speeds in order to investigate the wake region effects on the generation efficiency and fatigue loads. Their results showed that the helical path of the tip vortices was more pronounced at lower rotational speeds. Storey et al. [25] used a coupled LES/aero-elastic method to investigate the effect of multiple wind turbines operating with dynamic control actuation. A broad peak meandering frequency was indicated by the spectral analysis of the wake. They also showed that wake interactions have a significant effect at below-rated wind speeds, where high turbine thrust coefficients and large velocity deficit regions are observed. Zaghi et al. [26] analyzed the blockage effects for wind tunnel experiments on large-scale wind turbines. Their results showed that no unique equivalent section exists that allow both global coefficients and local flow features to be matched. 
Meyers and Meneveau [27] used LES to optimize the wind turbine spacing. They found optimal wind turbine spacing should be considered higher (15D) than conventional case (7D). Few studies have investigated power efficiency of downstream wind turbine when it operates in the shadow of upstream turbine $[28,29]$. Their results showed the efficiency is increased by increasing the lateral distance.

As mentioned above, most previous studies have focused on wind turbine performance, and the effect of wind turbine distance, yaw angles, etc. No study has yet been conducted in respect of the fluid flow details and their effects on wind farm performance. In other words, wind farm performance can be affected by turbulent structures as the flows around wind turbines are turbulent. The fatigue load of downstream wind turbines could be affected by turbulence intensity, as well as tip vortex frequency, tip vortices interferences and the dissipation distance of these vortices would be affected in the wake region. Therefore, the study of wake development, turbulence intensity, and turbulence structures are very important in order to gather comprehensive information to maximize the efficiency of wind farms. For this reason, this study aims to analyse turbulent structures which use the $\lambda_{c i}$ technique, and study the wind turbine's performance in different conditions. It is worth considering the effect of turbulent structures on wind turbine efficiency. Moreover, due to the conservation of angular momentum, flow is rotated in the opposite direction to the upstream rotor. In other words, flow in the wake region is rotated in the opposite direction of the blades [4]. Therefore, if the downstream turbine rotates in the opposite direction, more energy may be harvested. Hence, the effect of blade rotation direction on wind turbine efficiency must be investigated. In other words, the wind farm's efficiency is to be studied in relation to whether the wind turbines are rotated in the same direction or they are rotated in opposite directions.

The present work is based on the "Blind Test" workshop results [30, 31]. First, LES is performed around a single wind turbine. Two in-line wind turbines are then tested, whereby the downstream turbine is operated at different rotational speeds (co-rotating setup). In the next stage, LES is performed around a single turbine that rotates in a clockwise direction (when observed from the upstream side) at different rotational speeds. The blade of this turbine is created by mirroring the counter clockwise blade in order to rotate the turbine in a clockwise direction. Then, two in-line wind turbines with different configurations are tested (counter-rotating setup). Wake development, velocity changes, turbulence intensity, and swirling strength are studied behind the wind turbine in two configurations in order to obtain detailed information on fluid flow in the wake region.

\section{Numerical simulation}

\subsection{Wind tunnel and the model of wind turbines}

The "Blind Test" workshop (BT1) was held in Bergen, October 2011 (Fig.1a). The BT1 model had been extensively tested at NTNU. The "Blind Test 2" workshop (BT2) was organized by Norcowe and Nowitech in Trondheim, Norway in October 2012 and proved a more complicated model than BT1. Performance and wake development information were obtained for two similar turbines operating in line (Fig.1b). 
A single-wind turbine was used in the BT1 workshop, whereas two similar in-line wind turbines were used in BT2. The wind turbine in BT1 was the same as the downstream turbine in BT2. In both setups, the wind turbines were rotated in a counter-clockwise direction (when observed from the upstream side). The downstream wind turbine was rotated at a different rotational speed to BT2. The upstream wind turbine was similar to the downstream wind turbine, and had the same geometry, but a slightly different hub size led to somewhat different rotor diameters: $D_{1}=0.944 \mathrm{~m}$ while $D_{2}=0.894 \mathrm{~m}$. To build the blades with NREL S826, airfoil was used along the entire span of the blade. This profile was designed to achieve a high lift coefficient and low sensitivity to roughness [32]. The airfoil section is shown in Fig 2a. The NREL S826 wind turbine blade is shown in Fig. 2b (see [33] for further details). The wind tunnel in this study has a cross section of $\mathrm{L}_{X}=2.7 \mathrm{~m}$, a height of $\mathrm{L}_{\mathrm{Y}}=1.8 \mathrm{~m}$ and a total length of $\mathrm{L}_{\mathrm{Z}}=11.14 \mathrm{~m}$. The wind turbine is located $5 \mathrm{D}$ rotor diameters $\left(\mathrm{L}_{\mathrm{Z} 1}+\mathrm{L}_{\mathrm{Z2}}\right)$ downstream of the inlet section of the wind tunnel for single setup (Fig3.a). In both setups, the turbines have been centered in the wind tunnel. The upstream turbine is located $2 \mathrm{D}\left(\mathrm{L}_{\mathrm{Z1} 1}\right)$ rotor diameter from the inlet (Fig3.b). The distance between the two turbines is $3 \mathrm{D}\left(\mathrm{L}_{\mathrm{Z} 2}\right)$. The hub height of the wind turbine from the floor of the wind tunnel is $0.817 \mathrm{~mm}$ for both setups. The rotational directions of blades in different configurations are also seen in Fig3.

\subsection{Boundary conditions and computational mesh}

A uniform velocity of $10 \mathrm{~m} / \mathrm{s}$ is applied at the inlet. The turbulence intensity of the flow that enters the computational domain is $0.3 \%$. Turbulence intensity is defined as the ratio of standard deviation to the mean value of the wind speed.

$T I=\frac{\left\langle u^{2}\right\rangle^{1 / 2}}{U_{r e f}}$

At the outlet, the ambient pressure condition is applied. No slip condition is considered at the blades, towers, or walls of the wind tunnel. The tip speed ratio is $\lambda=6$ for the single setup. In the two in-line setup, the tip speed ratio of the upstream turbine is $\lambda_{1}=6$ and for the downstream turbine, it is $\lambda_{2}=4$. The downstream turbines operate at optimal conditions at this rotational speed (see [31] page 6). The tip speed ratio is defined as,

$\lambda=\Omega R / U_{r e f}$

"where $\Omega$ is the angular velocity, $R$ is the rotor radius, and $U_{r e f}$ is the velocity reference". The test section of the BT1 and BT2 workshops are simulated in this study. In other words, the computational domain has the same dimension as the aforementioned test sections. The computational domain consists of two moving and stationary zones (Fig 3). The moving zone consists of a cylindrical part and the wind turbine blades are located within the stationary zone, which includes the rest of the wind tunnel. Two cylindrical parts are considered for the two in-line setup. A sliding mesh technique is used for the moving zone. Unlike the Multi-Reference Frame (MRF) model, which consists of a one layer interface zone, the current model consists of a two-layer interface. 
A finite volume method is taken to discretize the governing equations. The convective terms are discretized using the Bounded Central Differencing scheme. The pressure term is computed by using the SIMPLE algorithm. The total number of cells in the numerical grid was $3.4 \times 10^{6}$ for the single setup and $4.6 \times 10^{6}$ for the two in-line setup. The time step value of $9.69 \times 10^{-4} \mathrm{sec}$ is considered. This time step is approximately equal to 7 degrees of blade rotation.

\subsection{Governing equation}

The velocity fields in a turbulent flow are three-dimensional, time-dependent and random. The main problem in turbulent flows is closure problem. The difference between modeling and simulation is on how to solve the closure problem. In simulation technique, scales of motion are resolved on length scales down to grid size for LES and to the Kolmogorov scale for Direct Numerical Simulation (DNS). In modeling all turbulence motions are time averaged and must somehow be modeled. LES is a separation between the larger three-dimensional unsteady turbulent motions and small scales. Large scale of motion is directly resolved whereas small scale motions are not explicitly resolved and must be modeled. LES computational expense lies between Reynolds-Stress Model (RSM) and Direct Numerical Simulation. RSM is limited to time averaged results. On the other hand, DNS cost is increased by the cube of Reynolds number. This is why the DNS method is inapplicable for high Reynolds number and complicated geometry. As the most engineering applications are at high Reynolds number and have complicate geometry. The LES method is strongly motivated. It is necessary to mention that more than 80 percent of turbulent kinetic energy is related to large scale motions that are directly studied using LES [34].

In the LES method the filtered continuum and incompressible Navier-Stokes are given as follows:

$$
\begin{aligned}
& \frac{\partial \bar{U}_{i}}{\partial x_{i}}=0 \\
& \frac{\partial \bar{U}_{j}}{\partial t}+\frac{\partial \overline{U_{i} U_{j}}}{\partial x_{i}}=v \frac{\partial^{2} \bar{U}_{j}}{\partial x_{i} \partial x_{i}}-\frac{1}{\rho} \frac{\partial \bar{p}}{\partial x_{j}}
\end{aligned}
$$

Here $\bar{U}_{i}$ is the resolved velocity, $\bar{p}(\mathrm{x}, \mathrm{t})$ is the filtered pressure field. Equation (4) differs from the Navier-Stokes equations because the filtered product, $\overline{U_{i} U_{j}}$, different than the product of the filtered velocities $\bar{U}_{i} \bar{U}_{j}$. The difference between $\bar{U}_{i} U_{j}$ and $\bar{U}_{i} \bar{U}_{j}$ is the residual-stress tensor that is defined by:

$\tau_{i j}^{R}=\overline{U_{i} U_{j}}-\bar{U}_{i} \bar{U}_{j}$

This tensor is analogous to the Reynolds stress tensor:

$\left\langle u_{i} u_{j}\right\rangle=\left\langle U_{i} U_{j}\right\rangle-\left\langle U_{i}\right\rangle\left\langle U_{j}\right\rangle$

The filtered momentum equation can be written as: 


$$
\frac{\partial \bar{U}_{j}}{\partial t}+\frac{\partial \bar{U}_{i} \bar{U}_{j}}{\partial x_{i}}=v \frac{\partial^{2} \bar{U}_{j}}{\partial x_{i} x_{i}}-\frac{1}{\rho} \frac{\partial \bar{p}}{\partial x_{j}}-\frac{\partial \tau_{i j}^{R}}{\partial x_{i}}
$$

Non- isotropic residual stress tensor is defined as follows:

$$
\tau_{i j}^{r}=\tau_{i j}^{R}-\frac{2}{3} k_{r} \delta_{i j}
$$

where $\delta_{i j}$ is Kronecker delta and $k_{r}$ is the residual kinetic energy and is defined as:

$$
k_{r}=\frac{1}{2} \tau_{i i}^{R}
$$

Isotropic residual stress appeared in the modified filtered pressure field:

$$
\bar{p}=\bar{p}+\frac{2}{3} \rho k_{r}
$$

Momentum equation can be rewritten as follows:

$$
\frac{\partial \bar{U}_{j}}{\partial t}+\frac{\partial \bar{U}_{i} \bar{U}_{j}}{\partial x_{i}}=v \frac{\partial^{2} \bar{U}_{j}}{\partial x_{i} \partial x_{i}}-\frac{1}{\rho} \frac{\partial \bar{P}}{\partial x_{j}}-\frac{\partial \tau_{i j}^{r}}{\partial x_{i}}
$$

\subsection{Residual-stress model}

Like RANS equations closure problem is also existed in the LES method. A model is needed for the anisotropic residual stress tensor $\tau_{i j}^{r}$ to somehow overcome this issue. The simplest model proposed by Smagorinsky [35]. The Residual stress tensor is related to the filtered rate of strain by linear eddyviscosity:

$$
\begin{aligned}
\tau_{i j}^{r} & =-2 v_{r} \bar{S}_{i j} \\
\bar{S}_{i j} & =\frac{1}{2}\left(\frac{\partial \bar{U}_{i}}{\partial x_{j}}+\frac{\partial \bar{U}_{j}}{\partial x_{i}}\right)
\end{aligned}
$$

Here $v_{r}(\mathrm{x}, \mathrm{t})$ is the eddy viscosity of the residual motions and $\bar{S}_{i j}$ is the filtered rate of strain. The eddy viscosity is modeled by analogy of the mixing-length hypothesis as below:

$$
\begin{aligned}
v_{r} & =\ell_{s}^{2} \bar{S} \\
& =\left(C_{S} \Delta\right)^{2} \bar{S}
\end{aligned}
$$

Here $\bar{S}$ is the characteristic filtered rate of strain, $\ell_{s}$ is the Smagorinsky length scale.Many residualstress models have been also proposed to overcome the closure problem. Germano et al. [36] proposed a dynamic model that utilized two different filters to determine an appropriate local value of the Smagorinsky coefficient, important modification and extensions for Germane model are provided by Lilly [37] and Meneveau et al [38] later. Other residual-stress models that based on gradients of the filtered velocity $\partial \bar{U}_{i} / \partial x_{j}$ or on the doubly filtered velocity $u$ have been also proposed. Often these models are used in combination with the Smagorinsky model, to form so-called mixed models, Bardina 
et al [39] and Clark et al [40]. In the current study Smagorinsky model with constant coefficient, $C_{S}=0.1$, are used for two setups.

\section{Results and Discussions}

\subsection{Validation}

In this section, the results of LES in a single turbine and two in-line turbines are compared with the experimental data and two different numerical simulations. The power coefficient for a single turbine (Fig4.a) and two in-line turbines (Fig4.b) is compared with the experimental data at different rotational speeds. The time-averaged velocity profile along the horizontal lines centered is compared at 1D and $5 \mathrm{D}$ in the downstream of a single turbine with the experimental data and two other numerical simulation results (Fig.5a, b). The results of the present work are consistent with the experimental data. The average difference from the experimental data is about $3 \%$, except on occasion in $5 \mathrm{D}$ where the difference is approximately $8 \%$. This discrepancy is also seen in the results of Hansen and Kono [30]. A similar study and comparison is performed for the two in-line setup (Fig.6).

\subsection{The comparison of pressure coefficients}

As previously highlighted, the clockwise blades are produced by mirroring the counter-clockwise blades. Hence, a single clockwise turbine is expected to have the same pressure coefficient as a single counter-clockwise turbine under similar conditions. Fig.7 shows the pressure coefficients at different rotational speeds for a single counter-clockwise turbine and a single clockwise turbine. The results revealed that pressure coefficients are almost identical at different rotational speeds in both setups. The two in-line turbines are then tested with two different configurations. The results show that the pressure coefficient is increased to about $4 \%$ for the downstream turbine in its counter-rotating configuration. In other words, the performance of the wind farm is increased without any change in the type of wind turbines or layout of the wind farm.

\subsection{Velocity profiles in the wake region}

In order to estimate the changes in the wake region as fluid moves downstream of the wind turbine, velocity profiles are compared at different locations behind the wind turbine for both a single wind turbine and two in-line wind turbines. A considerable portion of wind energy is harvested by the upstream wind turbine, so streamwise velocity is decreased and cross stream and span wise velocities are increased. Fig. 8 shows the time-averaged velocity profile along the horizontal lines centered at different locations behind the wind turbines for both setups. For both setups, the wake effect is reduced by increasing the downstream distance. The local minimum at the edge of the velocity profile could be associated with the tip vortices that are shed from each of the blades. These issues are seen in both setups. The velocity profile is not symmetrical for the two in-line setup as a result of the effect of the upstream wind turbine. 
At the center of the wind tunnel, where the hub is located, the local maximum is seen for both setups, because the lower energy at this location is harvested from the wind. For the two in-line turbines, the velocity deficit is higher than a single turbine at the hub location. This finding could be associated with the effect of the upstream turbine on the fluid flow that it is located in the path of the downstream turbine. The comparison of Fig. 8 (a) and Fig. 8 (b) shows that the wake effect in the two in-line setup is more intense than a single turbine, but the rate of wake recovery is faster than a single turbine. In the BT1 model, the researchers did not pay attention to the upper limit of the blockage ratio effect. In fact, this ratio should not exceed $10 \%$ [41], whereas it was approximately $13 \%$ for the BT1 model. Therefore, the streamwise velocity exceeds the reference velocity. If the blockage ratio is less than $10 \%$, the streamwise velocity exceeding the reference velocity should generally be negligible. A further decline in streamwise velocity in the swept area is seen for the two in-line turbines compared to a single turbine. This decline causes higher streamwise velocity near the wall for the two in-line turbines. Fig. 9 shows the cross stream velocity profile along a horizontal line centered at different locations behind the wind turbine for both setups. According to the law of conservation of momentum, when the velocity component is decreased in a streamwise direction, the velocity components are increased in the transverse direction. This condition is inversed by moving in a downstream direction. In fact, the streamwise velocity is increased, while the cross stream velocity is decreased by moving downstream. In the wake region, the cross stream velocity component deviates from the reference velocity $\left(\mathrm{V}_{\mathrm{y}}=0\right)$, because of the effect of the wind turbines in both setups. The deviation value in the two in-line turbines is higher than a single turbine (Fig.9a, b). Moreover, the deviation area in the span wise direction is different in the two setups. The deviation area ranges from $\mathrm{X} / \mathrm{R}=-1.2$ to 1.2 for a single turbine, whereas it ranges from $\mathrm{X} / \mathrm{R}=-1.5$ to 1.5 for the two in-line turbines. These differences could be associated with the effect of the two turbines on the fluid flow. On the other hand, the fluid flow is affected by the upstream turbine and is then affected by the downstream turbine once again, which means that the effect of the turbines on the fluid flow is intensified by the two in-line turbines.

Fig.10 shows the streamwise velocity profile along a vertical line centered at two locations behind the wind turbine for both setups. Moving in the downstream direction of the wind turbine causes the velocity profile to become smoother. This trend is more evident for the two in-line setup. The comparison between the two setups shows that the velocity deficit in the two in-line setup is more intense than a single wind turbine. The streamwise velocity is increased from zero close to the floor of wind tunnel to a maximum local value at $\mathrm{Y} / \mathrm{R}=-1.4$ for a single turbine. This location is the overlap point between the tower and impeller. The streamwise velocity is decreased to a minimum value of $\mathrm{Y} / \mathrm{R}=-1$, in which this point is at the middle of blade. Then, the streamwise velocity is increased from $\mathrm{Y} / \mathrm{R}=-1$ to 0 , where $\mathrm{Y} / \mathrm{R}=0$ is at the middle of blade in the upper part of the wind tunnel. It seems that the tip point of the blade extracts more energy from the wind than the root point of the blade. As the blade speed at the tip is much higher than the root, the energy extraction is affected by the blade speed. Therefore, the velocity is also affected when the wind energy varies. This trend is also seen for the two in-line turbines, but the velocity deficit is more intense at the tower location than for a single turbine, which can be caused by the effect of the upstream tower. The velocity deficit is more intense at the 
upper part of the wind tunnel for the two in-line setups, as a result of the wind energy harvested by the upstream rotor.

The characteristics of the wake region reveal why a counter-rotating configuration can harvest more wind energy than a co-rotating configuration under similar conditions. The rotation flow direction is a function of blade rotation direction. In a counter-rotating configuration, the flow field behind the upstream turbine has the same direction as the rotation direction of the downstream turbine. Therefore, when the flow field of the upstream turbine reaches the downstream turbine, more wind energy is harvested under similar conditions, compared to the co-rotating configuration.

Fig.11 shows the cross stream velocity profile along a horizontal line centered at two locations behind the wind turbine for both co-rotating and counter-rotating configurations. The wake of the downstream turbine penetrates the wake of the upstream turbine and produces this distribution shape. A portion of the cross stream velocity that is produced by the upstream turbine is absorbed by the downstream turbine.

Fig.12 shows the time-averaged streamwise velocity profile in the co-rotating and counter-rotating configurations. The development of streamwise velocity in the wake region is almost identical for both configurations. In other words, the higher efficiency of the counter-rotating configuration has not been influenced by the streamwise velocity, whereas the cross stream velocity has changed greatly. As a result, more power efficiency is associated with the lateral velocities. Therefore, the power performance is expected to increase when the space between the two turbines in the counter-rotating configuration decreases.

\subsection{Turbulence structures}

The downstream wind turbine is affected by the dynamic wind load of the upstream wind turbine. This wind load is due to fluid flow behavior. In other words, turbulence structures cause a significant dynamic wind load and vibrations in the downstream wind turbine. To optimize the efficiency of wind farms, and decrease the dynamic wind load, turbulence structures must be studied. For a horizontal-axis wind turbine, helical tip vortices are the main feature of this flow. The near wake region is affected by the number of blades, blade aerodynamics (such as attached or stalled flows), 3-D effects, and tip vortices [42]. Vorticity is not a suitable tool to reveal vortices, as it cannot differentiate between pure shearing motions and the actual swirling motions. Therefore, an accurate vortex extraction method is required to identify and track the vertical structures. Various tools and algorithms have been developed and proposed by many investigators for the identification of vortices [43-51]. In this study, the $\lambda_{\text {ci }}$ method [47] is used to extract the vortices.

The mathematical description and physical meaning of $\lambda_{\mathrm{ci}}$ are defined by Zhou et al. [47]. First of all, the local velocity field around a point can be expressed by the position vector $r$ in linear order as an equation

$u(\mathrm{r}+\delta \mathrm{r})=\mathrm{u}(\mathrm{r})+\mathrm{D} \delta \mathrm{r}+\mathrm{o}\left(\|\delta r\|^{2}\right)$

where $\mathrm{D}$ is the velocity gradient tensor $(\nabla u)$. Its characteristic equation is given by: 
$\lambda^{3}+P \lambda^{2}+Q \lambda+R=0$

where $Q=\frac{1}{2}\left[P^{2}-\right.$ trace $\left.(D D)\right], P=-\operatorname{trace}(D)=-$ div $(\boldsymbol{u})$ and $R=\frac{1}{3}\left[P^{3}+3 P Q-\operatorname{trace}(D D D)\right]$ are invariants of the velocity gradient tensor. The discriminant for this characteristic equation is:

$\Delta=\left(\frac{1}{2} \tilde{\mathrm{R}}\right)^{2}+\left(\frac{1}{3} \tilde{\mathrm{Q}}\right)^{3}$

Where $\tilde{R} \equiv R+2 / 27 P^{3}-\frac{1}{3} P Q$ and $\tilde{\mathrm{Q}} \equiv Q-1 / 3 P^{2}$. If $\Delta$ is positive, then the velocity gradient tensor has a real eigenvalue and a pair of conjugated complex eigenvalues. Furthermore, the rotation rate is quantified by $\lambda_{c i}$, and therefore Zhou and co-workers used the imaginary part of the complex eigenvalue pair as the local swirling strength of the vortex. If $\lambda_{c i}$ is equal to zero, then there is no swirling motion or local circular streamline. The two-dimensional form of the velocity gradient tensor can be written as

$\nabla u=\left[\begin{array}{ll}\partial u / \partial x & \partial u / \partial y \\ \partial v / \partial x & \partial v / \partial y\end{array}\right]$

The discriminant for this characteristic equation is

$\Delta=\operatorname{trace}(\nabla \mathrm{u})^{2}-4 \operatorname{det}(\nabla \mathrm{u})$

where $\lambda_{c i}$ is the imaginary part of the conjugate pair of the complex eigenvalues of $\nabla \mathbf{u}$ and it is not zero when $\Delta>0$. Swirling strength has not designated the sense of the rotation since it is defined as the imaginary part of the complex eigenvalue of the local velocity-gradient tensor. The sign of swirling strength specified according to the sign of the spanwise vorticity as follows:

$\lambda_{c i}=\frac{\omega_{x}}{\left|\omega_{x}\right|} \lambda_{c i}$

where $\omega_{x}$ is the instantaneous fluctuating spanwise vorticity. In the present study top-tip vortices in the upper part of the wind tunnel are presented with dark patch and root vortices with a light patch, while in the lower part of wind tunnel has opposite color.

Fig. 13 shows the vortices distribution in the wake region of a single turbine and two in-line wind turbines. Here, the effects of top-tip vortices are studied in more detail. The top-tip vortices disappear later than hub/root vortices. The effects of top-tip vortices are not visible after $1.5 \mathrm{D}$ in a streamwise direction. Lu and Porte '-Agel [52] showed that top-tip vortices are not seen after 2D in the wake flow of a wind turbine. For a single wind turbine, six or seven vortices are seen. Hu et al. [42] found that the gap between two adjacent vortices is a function of tip speed ratio. Top-tip vortices behind the downstream turbine take more area than a single turbine or upstream turbine. This could be associated with the fact that vortices are shed from the upstream turbine and are superimposed by the downstream turbine. In the wake of the downstream turbine, vortices are extended in the y direction, which could be due to the vortices extracted by the upstream wind turbine. These vortices interact with the downstream wind turbine and are probably superimposed. Bottom vortices are rapidly dissipated in the wake 
regions, which could result from the effect of tower and negligible velocity at the floor of the wind tunnel. It can also be seen that bottom vortices become far larger and persisted just as long in the two in-line wind turbines. The effect of the upstream turbine may intensify this structure.

\subsection{Turbulence intensity}

Turbulence intensity is defined as "a measure of fatigue loads on different parts of a wind turbine". Thus, the study of spatial distribution of turbulence intensity could be useful to minimize the effect of fatigue loads on downstream wind turbines. Fig.14 shows turbulence intensity along a horizontal line centered at one location after the inlet of a wind tunnel and at four locations behind the wind turbine in both setups.

The comparison of turbulence intensity before and after the wind turbines shows that turbulence intensity is increased in the downstream direction. Therefore, the downstream turbine experienced greater turbulence intensity than the upstream turbine. Turbulence intensity is also more intense for the two in-line setup than for a single wind turbine. This issue could be caused by the effect of the upstream turbine on turbulence intensity, which is superimposed onto the downstream turbine. An important feature in the near wake is the helical vortices that are shed from the blade tip. These vortices are the main source of noise and vibration of the blades. In both setups, around the $\mathrm{X} / \mathrm{R}= \pm 0.9$, two local maximum turbulence intensities are evident, which could result from the effect of tip vortices. The effect of these local maximum values is reduced by moving in the downstream direction. These local maximum values are dissipated at 7D in the two in-line setup. A local minimum turbulence intensity is obtained at the center of the impeller, which could even result from a blade speed that is near to zero at the root, and the vortices shed from the hub and root. In other words, less vibration caused less turbulence intensity at the root and a lower speed on the hub than at the tip locations. The intensity of this point in the two in-line setup is higher, because this setup is influenced by two consecutive turbines.

\section{Conclusion}

The present study focuses on the flow around a horizontal axis wind turbine. LES was employed in order to study the flow at the wake region for two different setups: single wind turbine (counter-clock wise and clockwise rotation), and two in-line wind turbines (co-rotating and counter-rotating configurations). The results are consistent with the experimental data reported in the literature. The wake region is affected by the number of turbines located on the upstream side. Wake extension is augmented by increasing the number of turbines. As one moves to the downstream side, the effect of the wake is decreased, but the wake recovery rate is faster due to the turbulence mixing in the two inline setup. The local minimum value in streamwise velocity at the edge of the swept area is the signature of tip vortices. The local maximum value at the hub location shows that lower energy is extracted at this location. In the wake region just behind the wind turbine, the cross stream velocity is deviated from the reference velocity in a ' $y$ ' direction. This deviation is intensified in the two in-line turbines. Cross stream velocity decreases as it moves in a downstream direction, while this trend is 
inversed for the streamwise velocity component. Wake study in the vertical direction shows that the span of blade from tip to middle harvests more wind energy than the middle to root in a single turbine. This phenomenon could result from the blade speed at the tip being far higher than the root speed. Similar behavior can also be seen in the two in-line turbine, but it is more intense. The comparisons between the counter-clockwise and clockwise rotation directions in a single turbine show that the pressure coefficients are almost the same at different rotational speeds. In the counter-rotating configuration, the pressure coefficient of the downstream turbine is increased by about $4 \%$. In the counter-rotating configuration, the rotation direction of the downstream turbine is the same as the upstream wake, which is why the downstream turbine in a counter-rotating configuration could harvest more energy than a co-rotating configuration. Streamwise velocity is almost identical at two locations in both configurations, but the cross stream velocity is greatly changed. In other words, the better performance of the downstream turbine in a counter-rotating configuration is due to the lateral velocities. The effect of vorticity and separated tip vortices in the near wake region is stronger than in the far wake region. As a result, the efficiency of the wind farm can be increased by decreasing the distance between two consecutive turbines in a counter-rotating configuration. This issue could be investigated in future work. In order to study turbulence structures, the $\lambda_{c i}$ method is employed to extract the turbulence structures and trace them in a downstream direction. Top-tip vortices are visible as far as $1.5 \mathrm{D}$. Top tip vortices are more extended behind the two consecutive turbines than a single turbine, which could result from the effect of upstream vortices being superimposed by the downstream turbine. Hub/root vortices travel a shorter distance than top vortices and dissipate sooner. The effects of bottom vortices are higher behind the two in-line turbines. The effect of upstream vortices may be superimposed. Turbulence intensity is also affected by the number of turbines located in the upstream side. Turbulence intensity is augmented by increasing the downstream distance. Local maximums at the edge of the blade tip are visible and are induced by tip vortices. A local minimum of turbulence intensity is seen at the hub location for both setups. This issue could be due to a blade speed that it is near to zero at the root and the vortices shed from the hub/root. The effect of this point is intensified in the two in-line setup. As aforementioned, the downstream turbines are affected by fluid flow and extracted turbulence structures via the upstream turbines, which is why investigating the fluid flow is important to increase the performance of wind farms.

\section{Acknowledgements}

The authors would like to acknowledge the Department of Energy and Process Engineering of the Norwegian University of Science and Technology for providing all the geometry data.

\section{References}

[1] Li Y, Paik KJ, Xing T, Carrica PM. Dynamic overset CFD simulations of wind turbine aerodynamics. Renew Energy 2012;37:285-98. doi:10.1016/j.renene.2011.06.029.

[2] Vermeer LJ, Sørensen JN, Crespo A. Wind turbine wake aerodynamics. Prog Aerosp Sci 2003;39:467-510. doi:10.1016/S0376-0421(03)00078-2.

[3] Massouh F, Dobrev I. Exploration of the vortex wake behind of wind turbine rotor. J Phys Conf Ser 2007;75:12036. doi:10.1088/1742-6596/75/1/012036. 
[4] Zhang W, Markfort CD, Porté-Agel F. Near-wake flow structure downwind of a wind turbine in a turbulent boundary layer. Exp Fluids 2012;52:1219-35. doi:10.1007/s00348-011-1250-8.

[5] Calif R, Schmitt FG. Modeling of atmospheric wind speed sequence using a lognormal continuous stochastic equation. J Wind Eng Ind Aerodyn 2012;109:1-8. doi:10.1016/j.jweia.2012.06.002.

[6] Calif R, Schmitt FG, Huang Y. Multifractal description of wind power fluctuations using arbitrary order Hilbert spectral analysis. Phys A Stat Mech Its Appl 2013;392:4106-20. doi:10.1016/j.physa.2013.04.038.

[7] Calif R, Schmitt FG. Multiscaling and joint multiscaling description of the atmospheric wind speed and the aggregate power output from a wind farm. Nonlin. Processes Geophys 2014;21:379-92. doi:10.5194/npg-21-3792014.

[8] Alfredsson PH, Dahlberg JA, Vermeulen PEJ. A comparison between predicted and measured data from wind turbine wakes. Wind Eng 1982; 6:149-55.

[9] Medici D, Alfredsson PH. Measurements on a wind turbine wake: 3D Effects and bluff body vortex shedding. Wind Energy2006; 9:219-36. doi: 10.1002/we.156.

[10] Crespo A, Hernandez J, Frandsen S. Survey of modeling methods for wind turbine wakes and wind farms. Wind Energy 2006; 2:1-24. doi: 10.1002/(SICI)1099-1824(199901/03)2:1<1::AID-WE16>3.0.CO;2-7.

[11] Troldborg N. Actuator line modeling of wind turbine wakes. Technical University of Denmark; 2008.

[12] Göçmen T, Laan P Van Der, Réthoré P, Diaz AP, Larsen GC, Ott S. Wind turbine wake models developed at the technical university of Denmark: A review. Renew Sustain Energy Rev 2016;60:752-69. doi:10.1016/j.rser.2016.01.113.

[13] van der Laan MP, Sørensen NN, Réthoré P-E, Mann J, Kelly MC, Troldborg N, et al. An improved k - $\epsilon$ model applied to a wind turbine wake in atmospheric turbulence 2016. doi:10.1002/we.1736

[14] Peña A, Réthoré P, Laan MP Van Der. On the application of the Jensen wake model using a turbulencedependent wake decay coefficient : the Sexbierum case 2016:763-76. doi:10.1002/we.

[15] Mo JO, Choudhry A, Arjomandi M, Kelso R, Lee YH. Effects of wind speed changes on wake instability of a wind turbine in a virtual wind tunnel using large eddy simulation. J Wind Eng Ind Aerodyn 2013;117:38-56. doi:10.1016/j.jweia.2013.03.007.

[16] Mo JO, Choudhry A, Arjomandi M, Lee YH. Large eddy simulation of the wind turbine wake characteristics in the numerical wind tunnel model. J Wind Eng Ind Aerodyn 2013;112:11-24. doi:10.1016/j.jweia.2012.09.002.

[17] Porté-Agel F, Wu Y-T, Lu H, Conzemius RJ. Large-eddy simulation of atmospheric boundary layer flow through wind turbines and wind farms. J Wind Eng Ind Aerodyn 2011;99:154-68. doi:10.1016/j.jweia.2011.01.011.

[18] Stevens RJAM, Gayme DF, Meneveau C. Large eddy simulation studies of the effects of alignment and wind farm length. J Renew Sustain Energy 2014;6:1-14. doi:10.1063/1.4869568.

[19] Norris S, Cater J. Wind turbine wake modelling using large eddy simulation. Proc ... 2010.

[20] Jimenez a, Crespo a, Migoya E, Garcia J. Advances in large-eddy simulation of a wind turbine wake. J Phys Conf Ser 2007;75:12041. doi:10.1088/1742-6596/75/1/012041.

[21] Li Q, Murata J, Endo M, Maeda T, Kamada Y. Experimental and numerical investigation of the effect of turbulent in fl ow on a Horizontal Axis Wind Turbine ( Part I : Power performance ). Energy 2016;113:713-22. doi:10.1016/j.energy.2016.06.138.

[22] Li Q, Kamada Y, Maeda T, Murata J, Nishida Y. Visualization of the flow field and aerodynamic force on a Horizontal Axis Wind Turbine in turbulent inflows. Energy 2016;111:57-67. doi:10.1016/j.energy.2016.05.098.

[23] Giahi MH, Jafarian Dehkordi A. Investigating the influence of dimensional scaling on aerodynamic characteristics of wind turbine using CFD simulation. Renew Energy 2016;97:162-8. doi:10.1016/j.renene.2016.05.059. 
[24] Zhong H, Du P, Tang F, Wang L. Lagrangian dynamic large-eddy simulation of wind turbine near wakes combined with an actuator line method. Appl Energy 2015;144:224-33. doi:10.1016/j.apenergy.2015.01.082.

[25] Storey RC, Cater JE, Norris SE. Large eddy simulation of turbine loading and performance in a wind farm. Renew Energy 2016;95:31-42. doi:10.1016/j.renene.2016.03.067.

[26] Zaghi S, Muscari R, Mascio A Di. Assessment of blockage effects in wind tunnel testing of wind turbines. J Wind Eng Ind Aerodyn 2016;154:1-9. doi:10.1016/j.jweia.2016.03.012.

[27] Meyers J, Meneveau C. Optimal turbine spacing in fully developed wind-farm boundary layers. Wind energy 2012; 15:305-17 .doi:10.1002/we.469.

[28] Chu CR, Chiang PH. Turbulence effects on the wake flow and power production of a horizontal-axis wind turbine. J Wind Eng Ind Aerodyn 2014;124:82-9. doi:10.1016/j.jweia.2013.11.001.

[29] Park J, Law KH. Layout optimization for maximizing wind farm power production using sequential convex programming. Appl Energy 2015;151:320-34. doi:10.1016/j.apenergy.2015.03.139.

[30] Krogstad PÅ, Eriksen PE. "Blind test" calculations of the performance and wake development for a model wind turbine. Renew Energy 2013;50:325-33. doi:10.1016/j.renene.2012.06.044.

[31] Pierella F, Krogstad PÅ, Sætran L. Blind Test 2 calculations for two in-line model wind turbines where the downstream turbine operates at various rotational speeds. Renew Energy 2014;70:62-77. doi:10.1016/j.renene.2014.03.034.

[32] Tangier JL, Somers DM. NREL airfoil families for HAWTs. Proc ... 1995.II

[33] Pierella F, Eriksen E, Sætran L. Invitation to the 2012 ” Blind test 2 "Workshop Calculations for two wind turbines in line 2012:1-11.

[34] Pope SB. Turbulent Flows 2000:770. doi:10.1179/030801801679395.

[35] Smagorinsky. General Circulation Experiments With the Primitive Equations. Mon. Weather Rev 1963; 91:99-64.

[36] Germano M, Piomelli U, Moin P, Cabot WH. A dynamic subgrid-scale eddy viscosity model. Phys. Fluids A Fluid Dyn 1991; 3:1760.

[37] Lilly DK. A Proposed Modification of the Germano-Subgrid-Scale Closure Method. Phys. Fluids A1992; 4:633-35.

[38] Meneveau C, Lund TS, Cabot WH. A Lagrangian dynamic subgrid-scale model of turbulence. J. of Fluid Mechanics 1996; 319:353-85. doi: 10.1017/S0022112096007379.

[39] Bardina J, Ferziger JH, Reynolds WC. Improved turbulence models based on large eddy simulation of homogeneous. incompressible turbulent flows 1983.

[40] Clark RA, Ferziger JH, Reynolds WC. Evaluation of subgrid- scale models using an accurately simulated turbulent flow. J. Fluid Mech1979; 91:1-16.

[41] Vries Ode. On the theory of the horizontal-axis wind turbine. J. Fluid Mech1983;15: 77-96. doi: 10.1146/annurev.fl.15.010183.000453

[42] Hu H, Yang Z, Sarkar P. Dynamic wind loads and wake characteristics of a wind turbine model in an atmospheric boundary layer wind. Exp Fluids 2012;52:1277-94. doi:10.1007/s00348-011-1253-5.

[43] Kim J. Evolution of a vortical structure associated with the bursting event in a channel flow. Proc1985.

[44] Jeong J, Hussain F. On the identification of a vortex. J. of Fluid Mechanics 2006; 285:69-94. doi:10.1017/S0022112095000462

[45] Perry AE, Chong ME, Cantwell BJ. A general classification of three-dimensional flow fields. J. Physics of Fluids 1990; 2:765-77. doi: 10.1063/1.857730.

[46] Hunt JCR, Wray AA, Moin P. Eddies, streams, and convergence zones in turbulent flows. Cent. Turbul. Res. Proc. Summer Progr1988; 1:193-208. 
[47] Zhou J, Adrian RJ, Balachandar S, Kendall TM. Mechanisms for generating coherent packets of hairpin vortices in channel flow. Journal of Fluid Mechanics 1999; 387: 353-96. doi:10.1017/S002211209900467X.

[48] Maciel Y, Shafiei Mayam MH. Hairpin structures in a turbulent boundary layer under stalled-airfoil-type flow conditions. Progress in Turbulence III 2008;131:199-202. doi:10.1007/978-3-642-02225-8_48

[49] Shafiei Mayam MH, Maciel Y. Hairpin structures in a turbulent boundary layer with strong adverse pressure gradient. International Symposium on turbulence and shear flow phenomena 2007.

[50] Shafiei Mayam MH, Maciel Y. Coherent structures in a turbulent boundary layer in stalled-airfoil0type flow conditions. AERO Conference and 54th Annual General Meeting 2007.

[51] Shafiei Mayam MH. Experimental study of the turbulence structures in a boundary layer subject to a strong adverse pressure gradient 2009.

[52] Lu H, Porte' -Agel F. Large-eddy simulation of a very large wind farm in a stable atmospheric boundary layer. J. of Phys Fluids 2011; 23:65-101.doi:10.1063/1.3589857. 


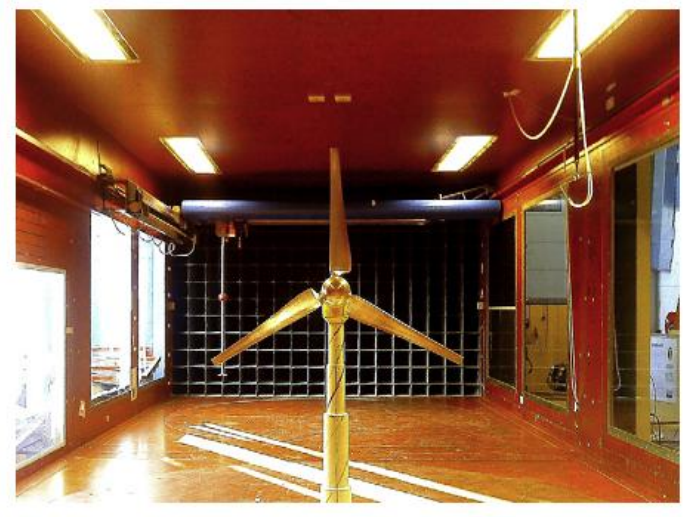

(a)

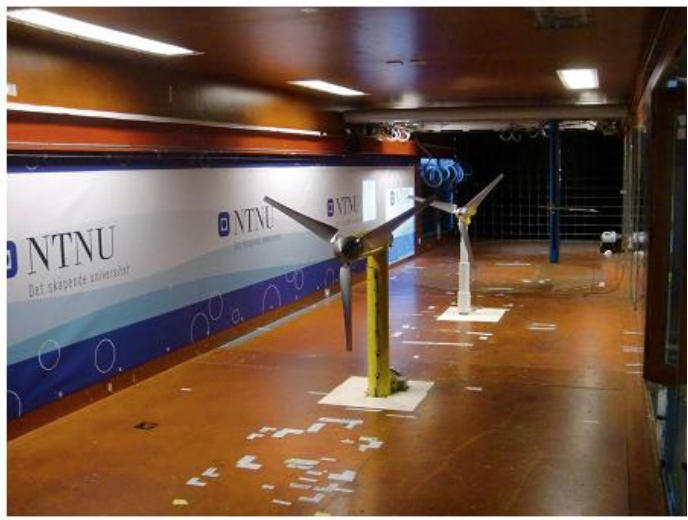

(b)

Fig. 1: (a).BT1 workshop [30]. (b) BT2 workshop [31].

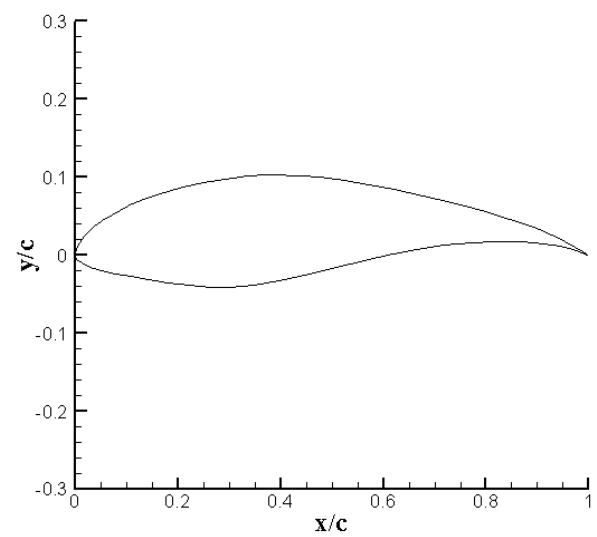

(a)

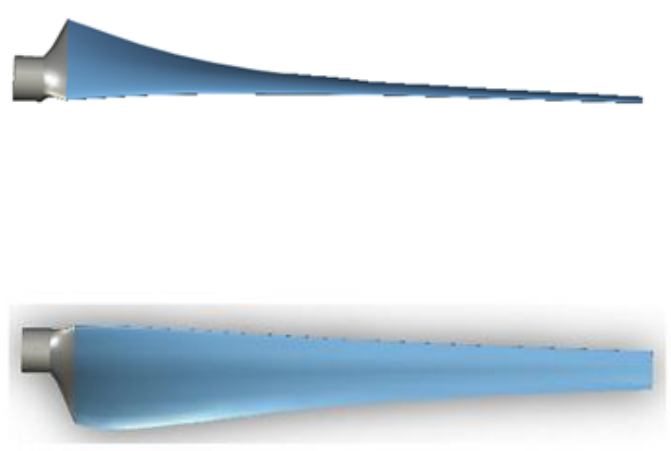

(b)

Fig. 2: (a) NREL S628 airfoil (b) blade in plane of rotation (top) and in the axial direction (down) [33].
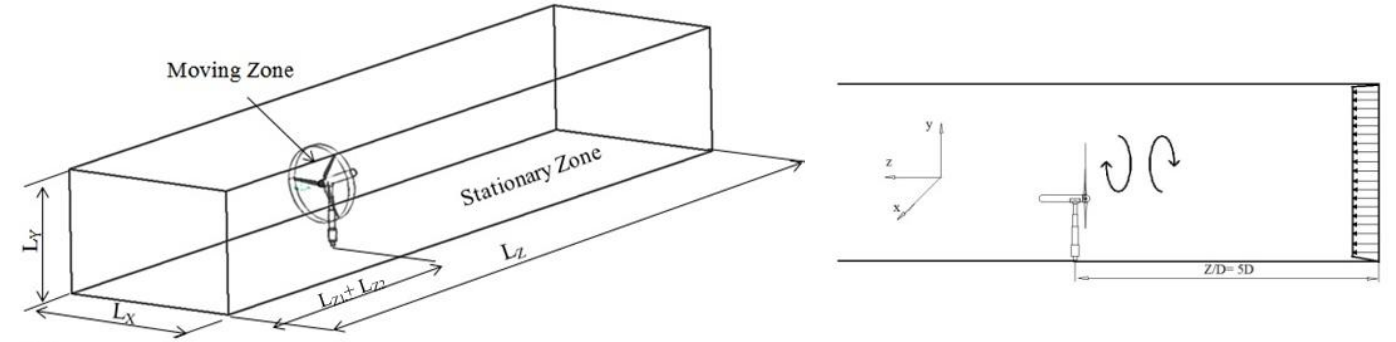<smiles>[Y]C([Y])[Z]</smiles>

(a)
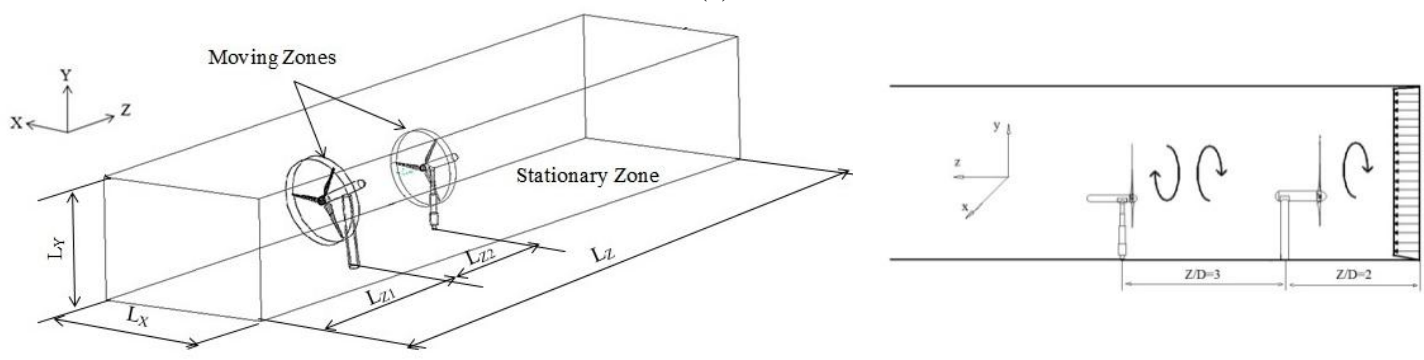

(b)

Fig. 3: Wind tunnel placement of the turbines (a) single setup (clockwise-rotating, counter-clockwise rotating), (b) two in line setup (co-rotating and counter-rotating configurations). 


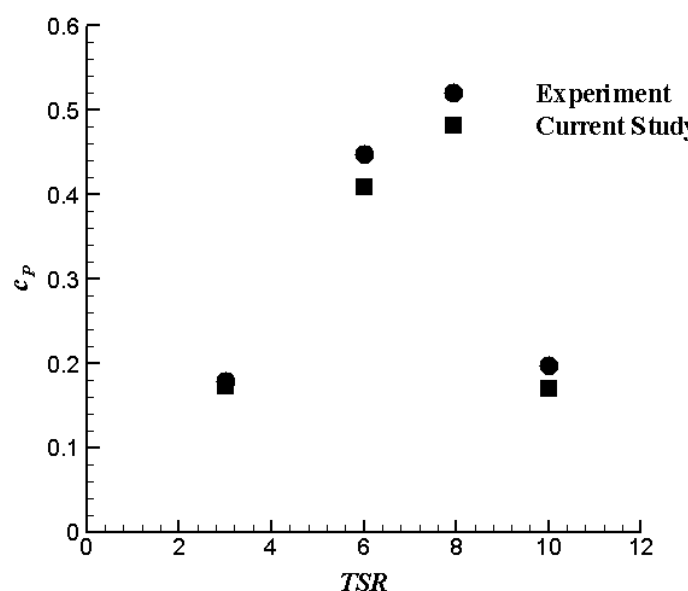

(a)

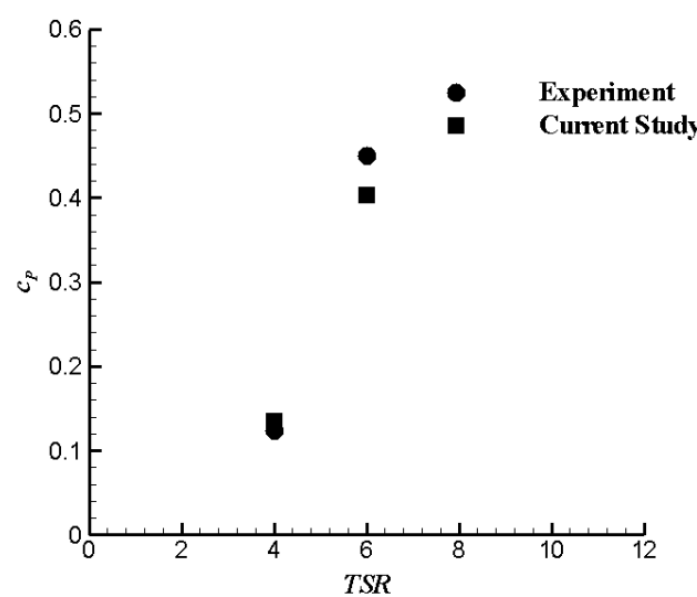

(b)

Fig. 4: $C_{P}$ coefficient of present work are compared by experimental data (a) single turbine, (b) two in line turbines

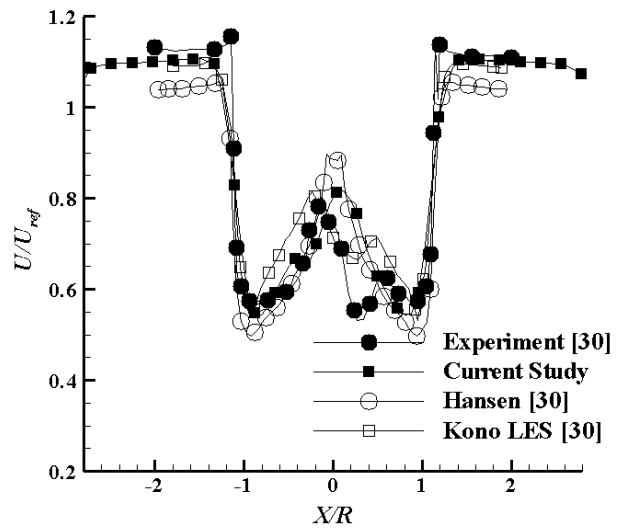

(a)

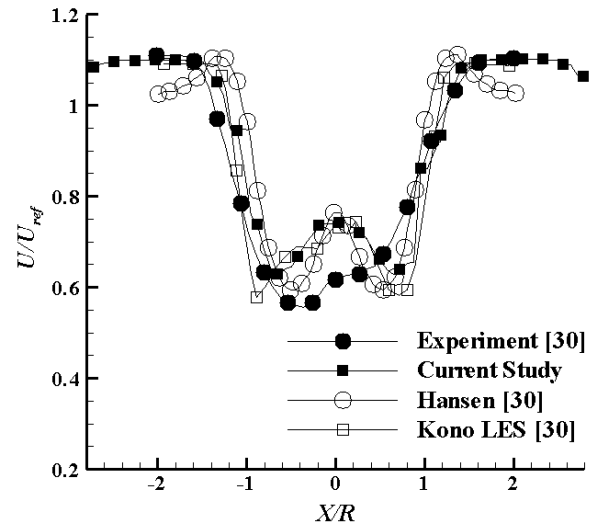

(b)

Fig. 5: Time averaged streamwise velocity profile along the horizontal lines centered at 1D (a) and 5D (b) in the downstream direction of a single turbine.

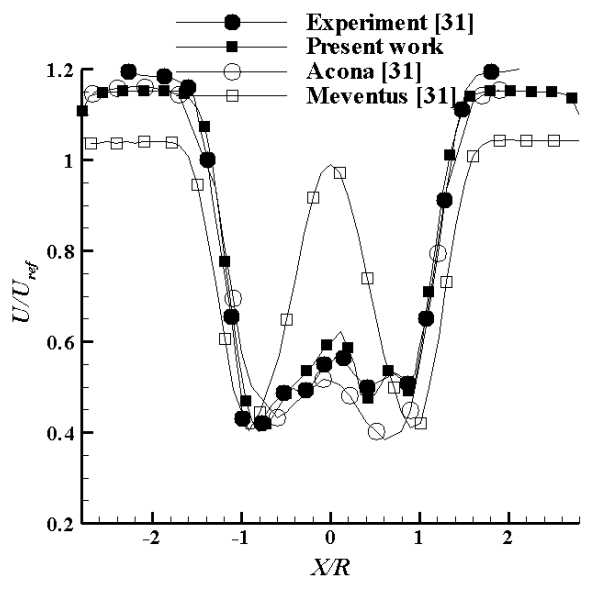

(a)

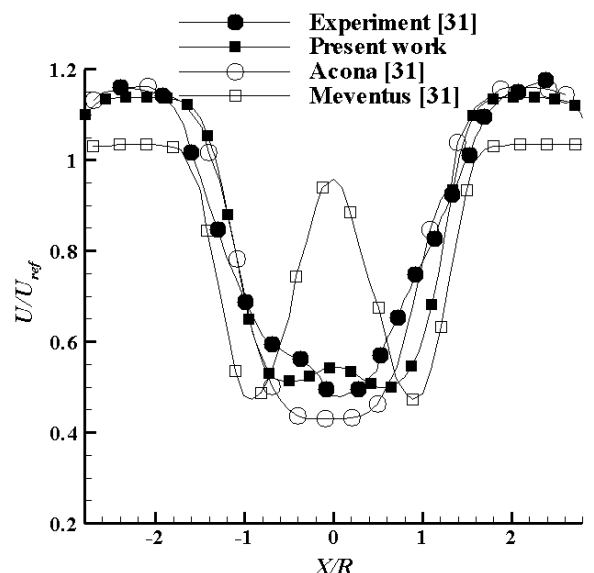

(b)

Fig. 6: Time averaged streamwise velocity profile along the horizontal lines centered at 1D (a) and 4D (b) in the downstream direction of a two in line turbines. 


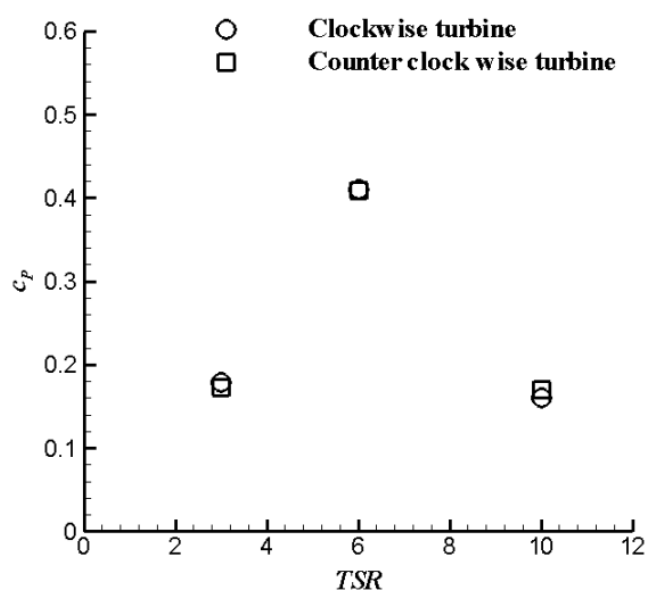

Fig. 7: The comparison of pressure coefficient of a clockwise and counter clockwise turbine.

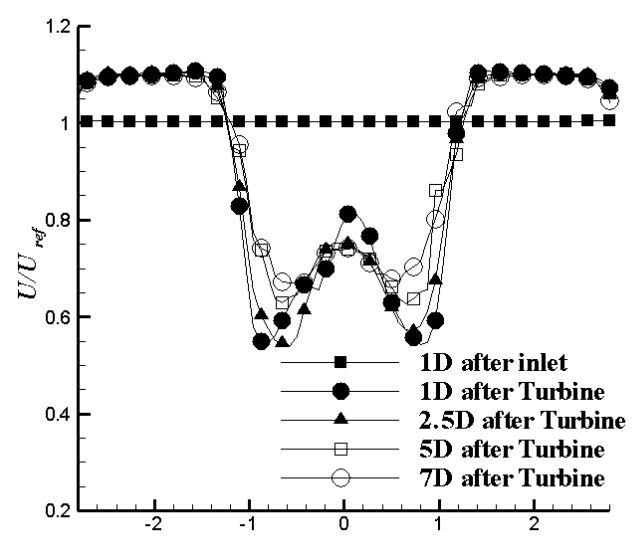

$X / R$

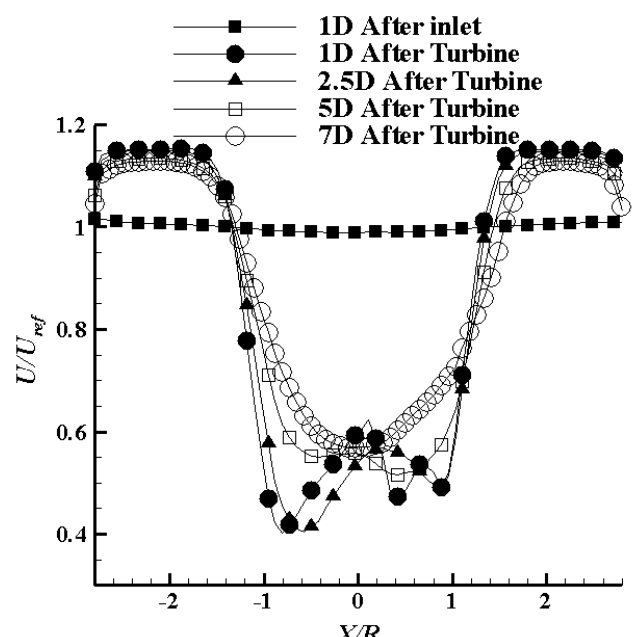

(b)

Fig. 8: Time averaged streamwise velocity profile along the horizontal lines centered at different locations (a) single turbine, (b) two in line turbines

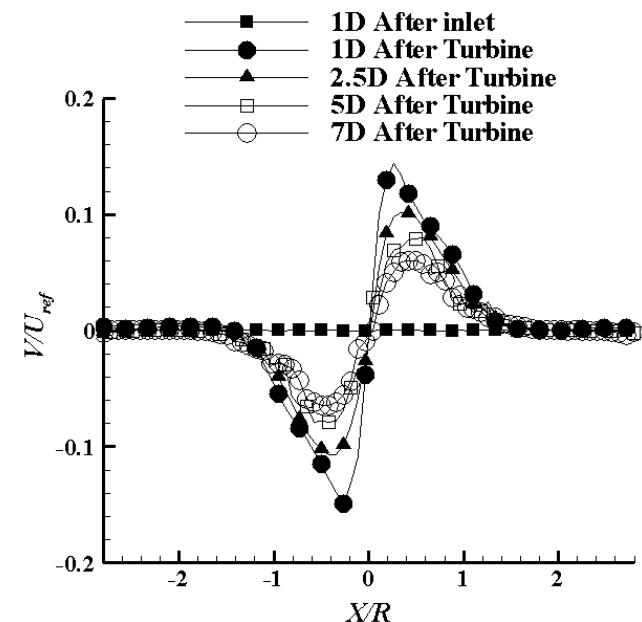

(a)

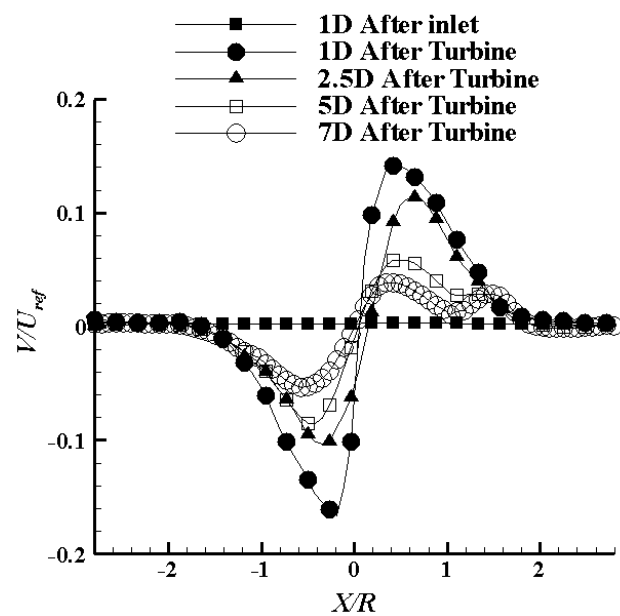

(b)

Fig. 9: Time averaged cross stream velocity profile along the horizontal lines centered at different locations (a) single turbine, (b) two in line turbines 


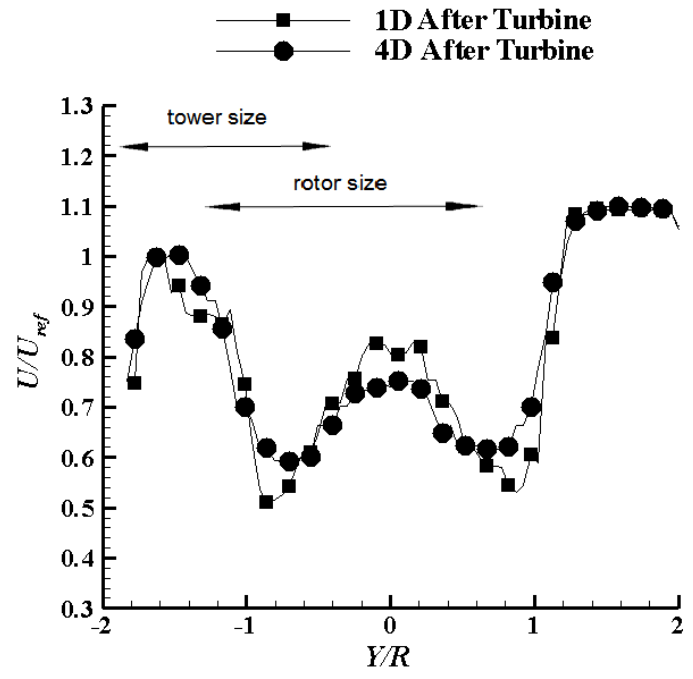

(a)

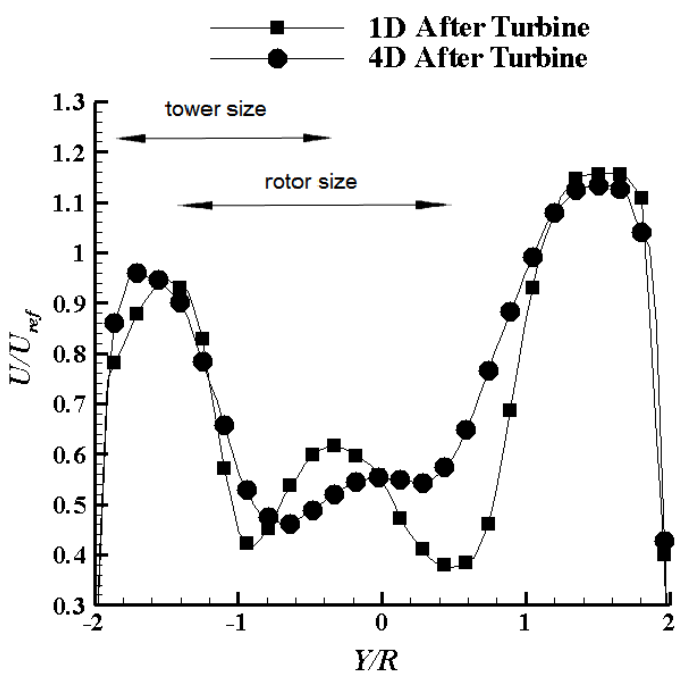

(b)

Fig. 10: Time averaged streamwise velocity profile along the vertical lines centered at different locations (a) single turbine, (b) two in line turbines

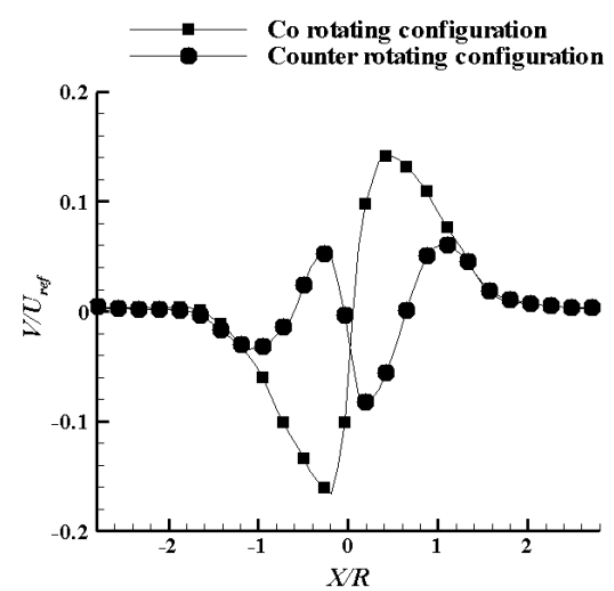

(a)

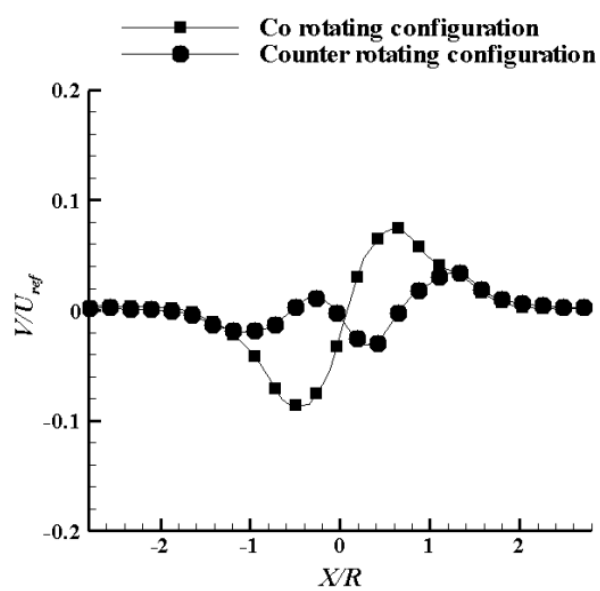

(b)

Fig. 11: Time averaged cross stream velocity profile along the horizontal lines at (a) 1D (b) 4D in the downstream direction of wind turbines in co- rotating and counter-rotating configurations. 


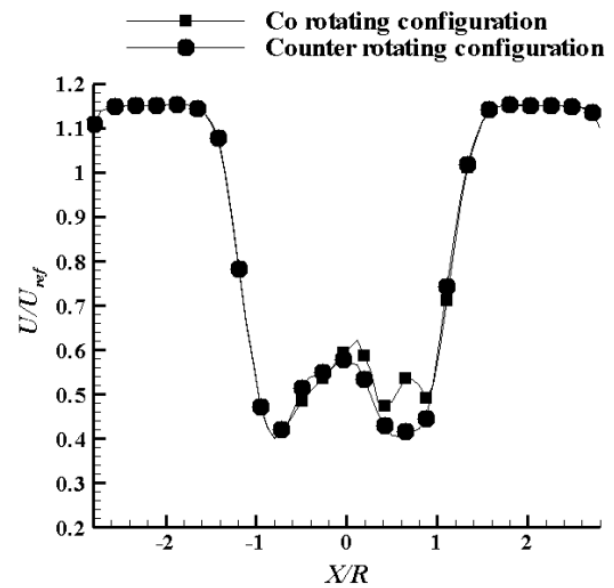

(a)

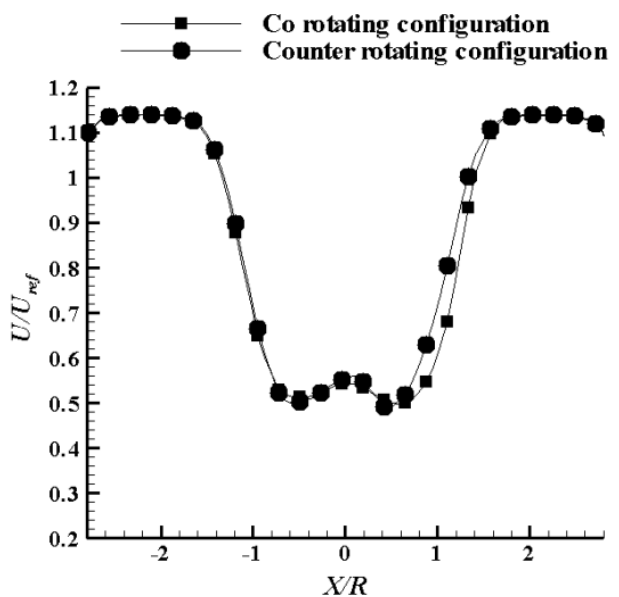

(b)

Fig. 12: Time averaged streamwise velocity profile along the horizontal lines at (a) 1D (b) 4D in the downstream direction of wind turbines in co- rotating and counter-rotating configurations

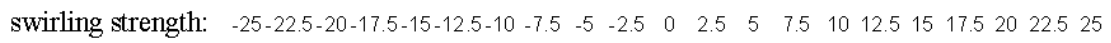

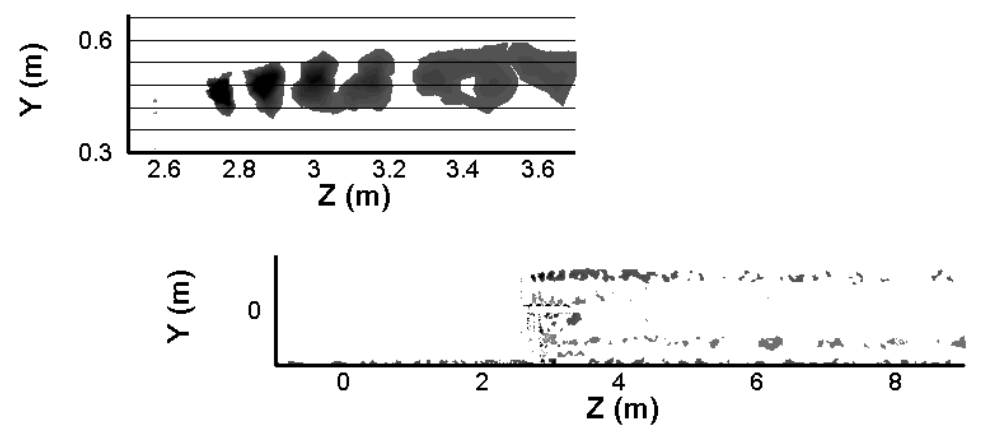

(a)

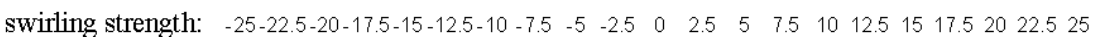
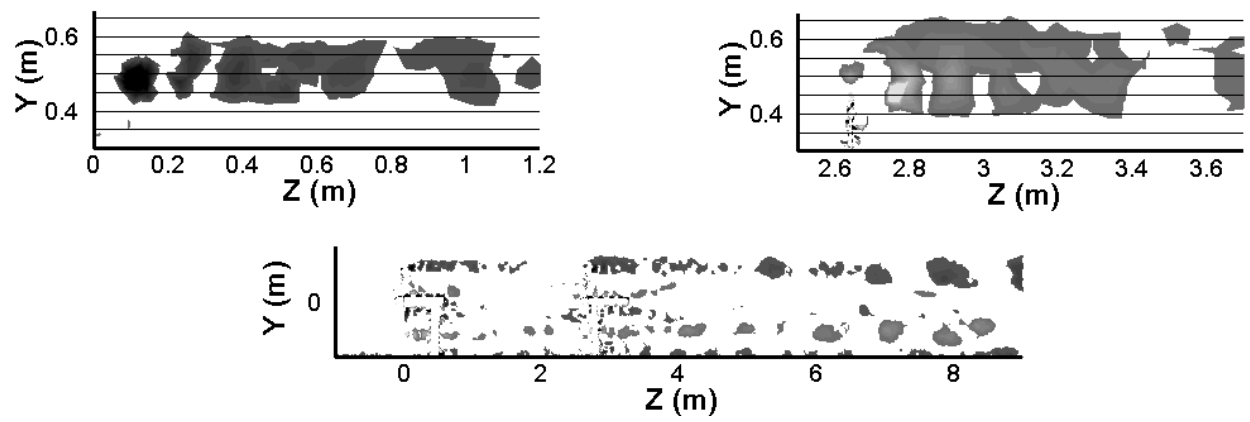

(b)

Fig 13: Vortex structures in the instantaneous velocity field (a) a single turbine (b) two in line turbines 


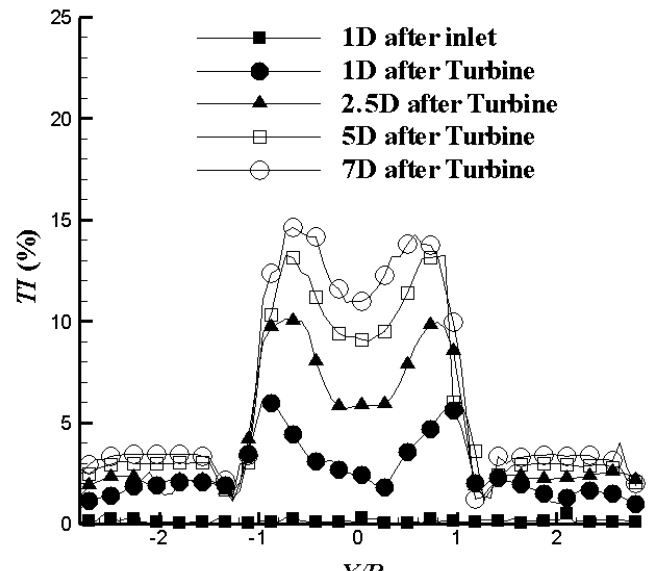

$X / R$

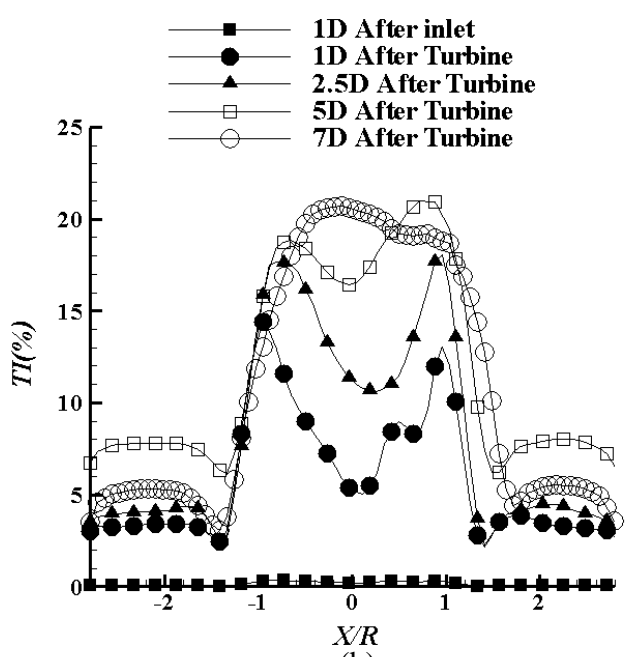

(b)

Fig. 14: Ensemble averaged streamwise turbulence intensity along the horizontal lines centered at different locations (a) a single turbine, (b) two in line turbines 